\title{
Flash Vacuum Thermolysis of 1,2,3-Thiadiazoles
}

\author{
Alan R. Katritzky, ${ }^{* a}{\text { Elizabeth L. Moyano, }{ }^{b} \text { Gloria Yranzo }}^{b}$ and Sandeep K. Singh ${ }^{a}$ \\ ${ }^{a}$ Center for Heterocyclic Compounds, Department of Chemistry, University of Florida, \\ Gainesville, FL 32611-7200, USA \\ ${ }^{b}$ INFIQC- Departamento de Química Orgánica, Facultad de Ciencias Químicas, Universidad \\ Nacional de Córdoba, Córdoba, Argentina \\ E-mail: Katritzky@chem.ufl.edu, yranzogi@dqo.fcq.unc.edu.ar
}

\section{Dedicated to Professor Oleg N. Chupakhin on the occasion of his $70^{\text {th }}$ birthday}

(received 14 Oct 04; accepted 14 Jan 05; published on the web 21 Jan 05)

\begin{abstract}
A series of 5-alkyl-, 5-aryl-, 5-aryloxy-, 5-arylthio- and 5-benzotriazolyl-4-phenyl-1,2,3thiadiazoles were subjected to flash vacuum thermolysis. Depending on the 5-substituent, the products were 1,4-dithiins, benzodithiins, benzothiophenes, disulfides or benzimidazoliumides.
\end{abstract}

Keywords: 1,2,3-Thiadiazoles, flash vacuum thermolysis, diradicals

\section{Introduction}

Thermal and photochemical reactions of 1,2,3-thiadiazoles (A) have been the subject of many investigations. ${ }^{1}$ Elimination of nitrogen leads to various intermediates (B) including: 1,3diradicals $\mathbf{i}$ and iii, thioacyl carbene iv and antiaromatic thiirene ii. Migration of $\mathrm{R}^{1}$ or $\mathrm{R}^{2}$ eventually leads to a thioketene $\mathbf{v}$ via Wolff-rearrangement (Scheme 1). The identification and trapping of intermediates i-iv has received much attention. Formation of 1,3-diradicals (i, iii) was evidenced by $\mathrm{ESR}^{2}$ and thiirenes (ii) were characterized by IR spectroscopy upon irradiating 1,2,3-thiadiazole in an Ar-matrix at $8 \mathrm{~K}$. ${ }^{3}$ Thioketenes (v) have been prepared and isolated by the application of the flash-vacuum thermolysis (FVT) technique. ${ }^{4-6}$

Previously identified products of thermolysis or photolysis of thiadiazoles include alkynes (C), thiophenes (D), 1,3-dithiafulvenes (E), and 1,2- or 1,4-dithiins (F) (Scheme 1). ${ }^{7}$ 1,2,3Thiadiazoles unsubstituted at position-5 or with a -Cl, -OAr or -SAr substituent undergo loss of $\mathrm{N}_{2}$ in the presence of a base to give alkynethiolates and subsequent quenching with alkyl- or acyl halides provides easy access to alkynylthioethers. ${ }^{8}$ 4,5-Disubstituted-1,2,3-thiadiazoles often provide stable thioketenes and are useful candidates to study the formation of rearrangement products. We have earlier reported base-promoted thermolysis of 4-aryl-5-heteroaryl/alkyl 
substituted 1,2,3-thiadiazoles. ${ }^{9}$ We now report the flash-vacuum thermolysis of 5-alkyl-, 5aryloxy-, 5-arylthio- and 5-benzotriazolyl-4-phenyl-1,2,3-thiadiazoles.

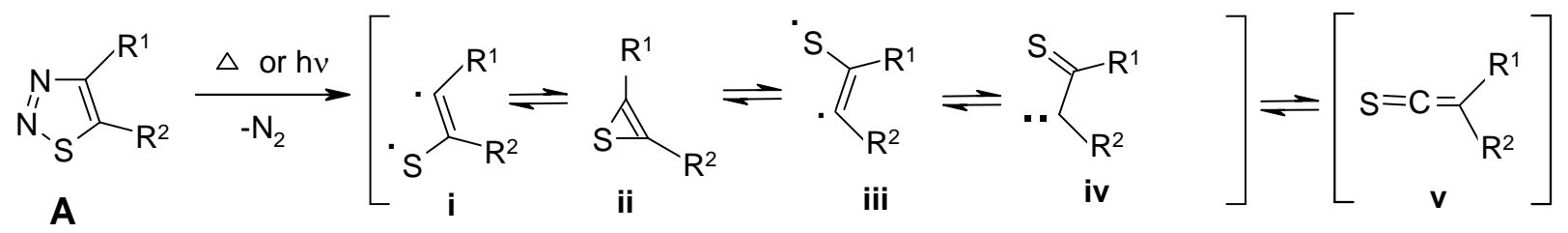

B

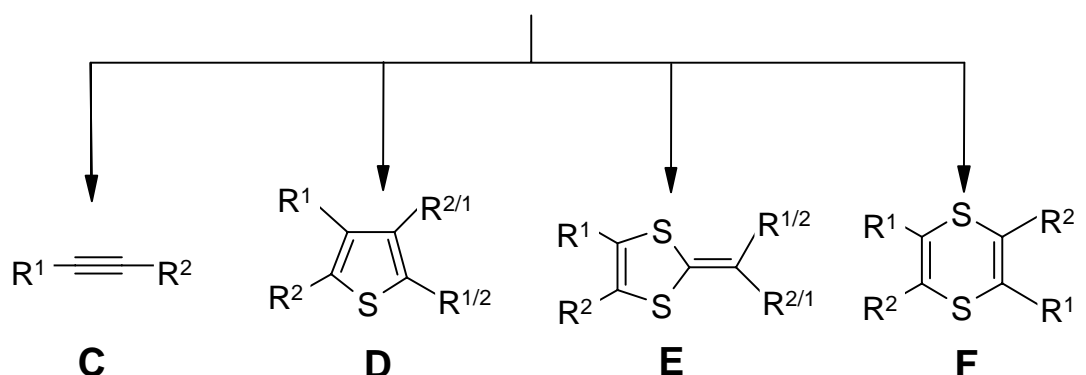

C

D

$E$

$\mathbf{F}$

\section{Scheme 1}

\section{Results and Discussion}

1,2,3-Thiadiazoles $\mathbf{1}, \mathbf{2}$, 8a,b, 11a-c and $\mathbf{1 7}$ were prepared using published procedures. ${ }^{9 \mathrm{~b}-\mathrm{d}}$ Flash vacuum thermolysis was carried out using a conventional flow system.

FVT of 5(4)-alkyl-4(5)-phenyl-1,2,3-thiadiazoles (1,2). Thermolysis of 4-phenyl-5-propyl1,2,3-thiadiazole (1) was carried out between $280-340^{\circ} \mathrm{C}$. In this case, at $340{ }^{\circ} \mathrm{C}$ the starting material was completely converted to dithiins $\mathbf{3}$ and $\mathbf{4}$ and trimer $\mathbf{5}$ as the main reaction products (Scheme 2). Minor products in the volatile fraction were 1,2,3-thiadiazole, acrylonitrile, 1phenyl-1-butyne, benzene and toluene. Thus, thiadiazole 1 loses nitrogen to afford 1,3-diradical 1(i), which is in equilibrium with thiirene 1(ii) and diradical 1(iii). Coupling of diradical intermediates 1(i) and 1(iii) gives dithiins 3 and 4 . The polymer 5 probably arises from the 1phenylpent-2-ene-1-thione formed from the diradical 1(iii) or carbene 1(iv). After several purifications a trimer $5(\mathbf{a})(\mathrm{n}=3)$ was isolated from the polymeric mixture.

FVT of 4-benzyl-5-phenyl-1,2,3-thiadiazole (2) was carried out between 300-400 ${ }^{\circ} \mathrm{C}$. After purification of the crude mixture, an isomeric mixture of benzothiines 6 and 7 was isolated. Benzothiines may arise from the expected nitrogen extrusion, when the diradical intermediate 2(i) undergoes two different reactions: insertion of the sulfur-centered radical into a $\mathrm{C}-\mathrm{H}$ bond followed by a $[1,3]$ hydrogen shift to give the product $\mathbf{6}$, or isomerization to thioketene 2(v), which cyclizes to give the isomer 7. No thermal isomerization of dithiins takes place under FVT conditions as evidenced by FVT of 6 from $300-350{ }^{\circ} \mathrm{C}$ when no formation of dithiin 7 was detected. 

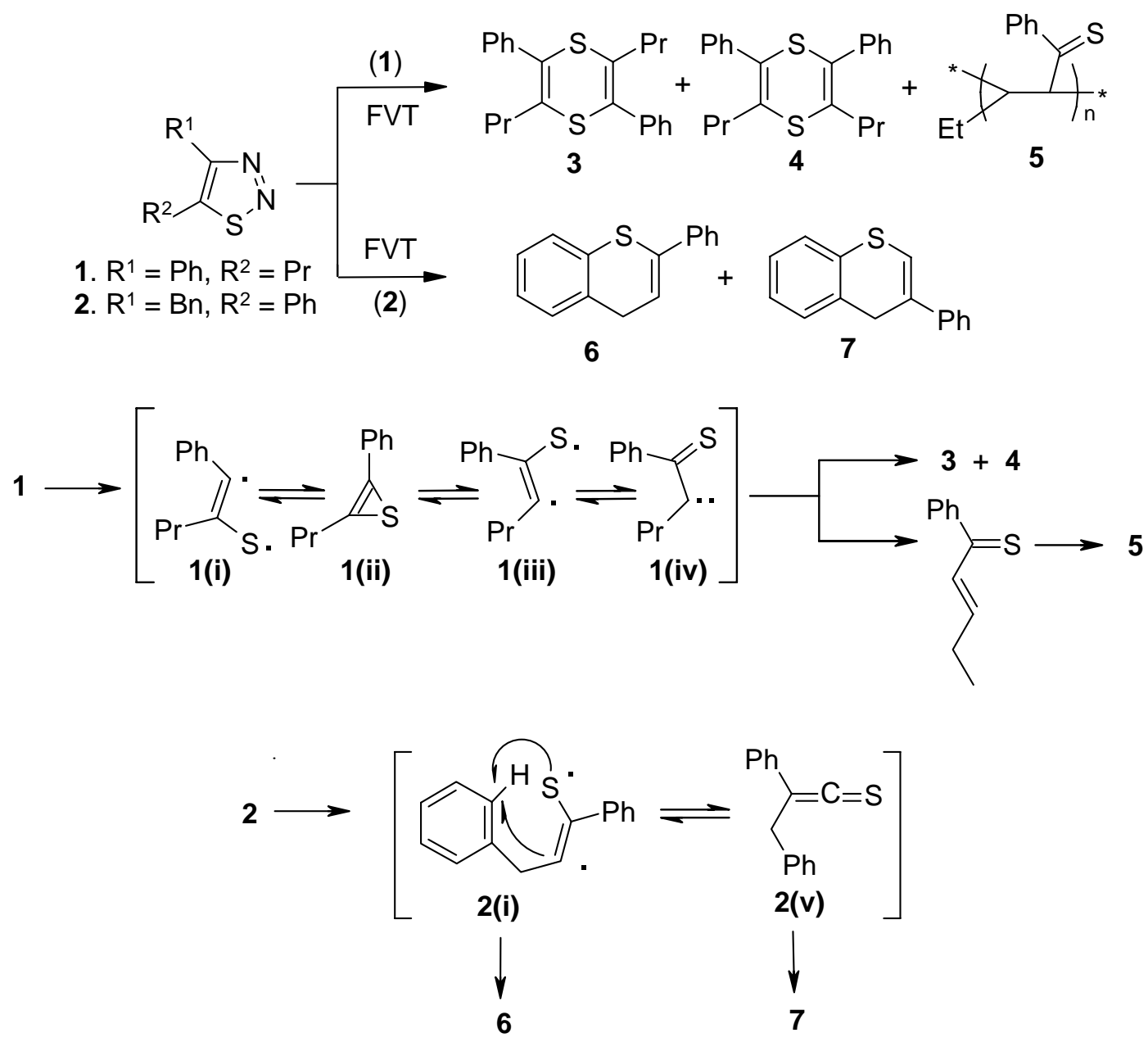

FVT of 1. $\mathrm{R}^{1}=\mathrm{Ph}, \mathrm{R}^{2}=\mathrm{Pr}$

\begin{tabular}{ccccc}
\hline $\mathrm{T}\left({ }^{\circ} \mathrm{C}\right)$ & $\% \mathbf{1}$ & $\mathbf{3 / 4} \mathrm{Y}(\%)$ & $\mathbf{5} \mathrm{Y}(\%)$ & others $\mathrm{Y}(\%)^{\mathrm{a}}$ \\
\hline 280 & 88 & - & - & 12 \\
300 & 45 & 8 & 32 & 15 \\
320 & 5 & 17 & 68 & 10 \\
340 & - & 15 & 70 & 15
\end{tabular}

aMinor products: 1,2,3-thiadiazole, 1-phenyl-1-butyne, toluene, acrylonitrile and benzene.

FVT of 2. $\mathrm{R}^{1}=\mathrm{Bn}, \mathrm{R}^{2}=\mathrm{Ph}$

\begin{tabular}{lccc}
\hline $\mathrm{T}\left({ }^{\circ} \mathrm{C}\right)$ & $2 \mathrm{Y}(\%)$ & $6 / 7 \mathrm{Y}(\%)$ & others $\mathrm{Y}(\%)^{a}$ \\
\hline 300 & 48 & 5 & 47 \\
325 & 31 & 19 & 50 \\
350 & 3 & 28 & 69 \\
400 & - & 22 & 78
\end{tabular}

aMinor products: 3-phenylindene, 1,3-diphenylpropyne, 1,1'-(2-cyclopropene-1-ylidene)bisbenzene.

\section{Scheme 2}


FVT of 5-aryloxy-4-phenyl-1,2,3-thiadiazoles (8). FVT of 5-(4-methylphenoxy)-4-phenyl1,2,3-thiadiazole (8a) was carried out between $170-250{ }^{\circ} \mathrm{C}$. In the reaction mixture, 2-(4methylphenoxy)benzothiophene (9a) and p-cresol (10a) were detected as the major products (Scheme 3). Formation of benzothiophene 9a can be explained by the formation of diradical 8(i), which then rearranges via insertion of the sulfur-centered radical into the $\mathrm{C}-\mathrm{H}$ bond of the phenyl ring. $p$-Cresol may be formed by a competitive radical reaction from the starting material when the $\mathrm{C}-\mathrm{O}$ bond is broken under thermal conditions. The presence of radicals was confirmed by formation of dibenzyl in reactions where toluene was used as a carrier gas.

Similarly, FVT of 5-(4-methoxyphenoxy)-4-phenyl-1,2,3-thiadiazole (8b) between 150-200 ${ }^{\circ} \mathrm{C}$ gave 2-(4-methoxyphenoxy)benzothiophene (9b) and 4-methoxyphenol (10b) as the principal products (Scheme 3).

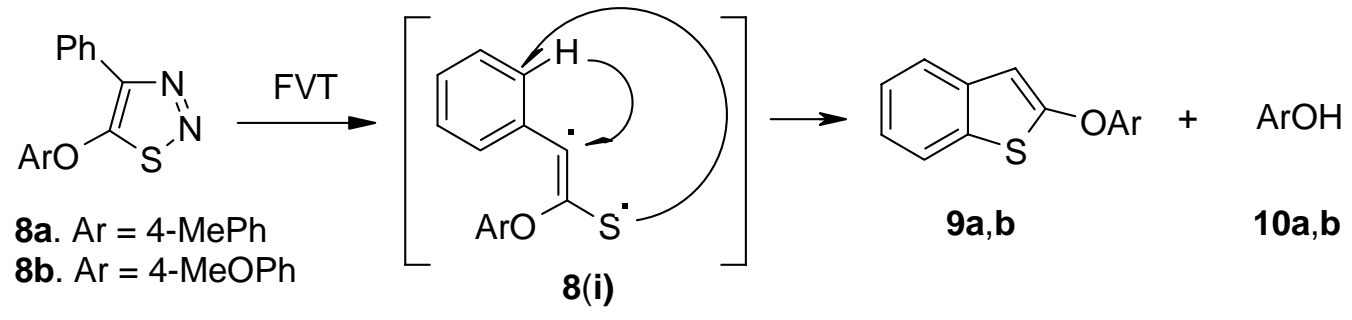

FVT of 8a. $\mathrm{R}=4-\mathrm{MePh}$

\begin{tabular}{ccccc}
\hline $\mathrm{T}\left({ }^{\circ} \mathrm{C}\right)$ & \%8a & $\mathbf{9 a} \mathrm{Y}(\%)$ & $\mathbf{1 0 a} \mathrm{Y}(\%)$ & others $\mathrm{Y}(\%)^{\mathrm{a}}$ \\
\hline 170 & 51 & 15 & 32 & 2 \\
200 & 15 & 39 & 40 & 6 \\
230 & 11 & 49 & 33 & 7 \\
250 & - & 65 & 26 & 9 \\
\multicolumn{2}{c}{ Unidentified products. } & & & \\
\hline
\end{tabular}

FVT of 8 b. $\mathrm{R}=4-\mathrm{MeOPh}$

\begin{tabular}{lcccc}
\hline $\mathrm{T}\left({ }^{\circ} \mathrm{C}\right)$ & $\% \mathbf{8 b}$ & $\mathbf{9 b} \mathrm{Y}(\%)$ & $\mathbf{1 0 b} \mathrm{Y}(\%)$ & others $\mathrm{Y}(\%)^{\mathbf{a}}$ \\
\hline 150 & 39 & 25 & 32 & 4 \\
180 & 28 & 31 & 36 & 5 \\
200 & 2 & 41 & 50 & 7 \\
\multicolumn{2}{c}{ Unidentified products. } & & & \\
\hline
\end{tabular}

\section{Scheme 3}

FVT of 4-phenyl-5-thioaryl-1,2,3-thiadiazoles (11). FVT of 5-[(4-methylphenyl)thio]-4phenyl-1,2,3-thiadiazole (11a) was carried out between $210-250^{\circ} \mathrm{C}$. This thiadiazole was very reactive under FVT conditions and a complex mixture of products was obtained. Dithiin 12a, and sulfides 14a and 15a were the main products (Scheme 4). 
<smiles>[X]c1cc([X])c(Sc2snnc2-c2ccccc2)cc1[X]</smiles><smiles>[Y]c1cc([X])c2c(c1[Z])C(c1ccccc1)C(=S)S2</smiles>

FVT of 11a. $X=Z=H, Y=M e$

\begin{tabular}{lcrrrr}
\hline $\mathrm{T}\left({ }^{\circ} \mathrm{C}\right)$ & \%11a & 12a $\mathrm{Y}(\%)$ & 14a $\mathrm{Y}(\%)$ & 15a $\mathrm{Y}(\%)$ & others $\mathrm{Y}(\%)^{\mathbf{a}}$ \\
\hline 210 & 80 & 6 & 5 & 5 & 4 \\
230 & 65 & 11 & 9 & 10 & 7 \\
250 & 35 & 26 & 12 & 18 & 9 \\
\multicolumn{7}{l}{ anidentified products. } & & &
\end{tabular}

FVT of 11b. $X=\mathrm{Z}=\mathrm{H}, \mathrm{Y}=\mathrm{Cl}$

\begin{tabular}{rrrrrrr}
\hline $\mathrm{T}\left({ }^{\circ} \mathrm{C}\right)$ & \%11b & 12b $\mathrm{Y}(\%)$ & $\mathbf{1 3 b} \mathrm{Y}(\%)$ & $\mathbf{1 4 b} \mathrm{Y}(\%)$ & $\mathbf{1 5 b} \mathrm{Y}(\%)$ & others $\mathrm{Y}(\%)^{\mathbf{a}}$ \\
\hline 210 & 58 & 13 & 5 & 5 & 10 & 9 \\
230 & 41 & 22 & 8 & 10 & 13 & 6 \\
250 & 32 & 25 & 4 & 15 & 16 & 8 \\
\multicolumn{2}{l}{ a Unidentified products. } \\
\hline
\end{tabular}

FVT of 11c. $X=Z=M e, Y=H$

\begin{tabular}{cccccc}
\hline $\mathrm{T}\left({ }^{\circ} \mathrm{C}\right)$ & \%11c & 12c $\mathrm{Y}(\%)$ & 13c $\mathrm{Y}(\%)$ & 14c $\mathrm{Y}(\%)$ & others $\mathrm{Y}(\%)^{\mathrm{a}}$ \\
\hline 250 & 42 & 25 & 19 & 6 & 8 \\
270 & 17 & 26 & 38 & 9 & 10 \\
300 & - & 26 & 48 & 15 & 11 \\
\multicolumn{7}{l}{ aMinor products: } & 2,5-dimethylthiophenol and 15c. \\
\hline
\end{tabular}

\section{Scheme 4}


FVT of 5-[(4-chlorophenyl)thio]- 4-phenyl-1,2,3-thiadiazole (11b) was carried out between 210-250 ${ }^{\circ} \mathrm{C}$. Dithiin 12b, benzothiophene 13b, and disulfides $\mathbf{1 4 b}$ and $\mathbf{1 5 b}$ were isolated as the main products from the pyrolysate (Scheme 4).

FVT of 4-phenyl-5-[(2,5-dimethylphenyl)thio]-1,2,3-thiadiazole (11c) was carried out between $250-300{ }^{\circ} \mathrm{C}$. Thiadiazole 11c was completely converted to products at $300{ }^{\circ} \mathrm{C}$. At lower temperatures $\left(250^{\circ} \mathrm{C}\right)$ dithiin 12c and benzothiophene 13c are the main products (Scheme 4). Dithiin 12c and benzothiophene (13c) are formed by nitrogen extrusion and rearrangement of diradical intermediates. The pyrolysates at $250-300{ }^{\circ} \mathrm{C}$ showed a deep green color, which disappeared at high temperatures. The coloration changed to yellow when the crude reaction mixture was kept at RT for several hours. When the ${ }^{1} \mathrm{H}-\mathrm{NMR}$ spectrum was run immediately after the reaction, a typical signal at $4.52 \mathrm{ppm}$ was detected, corresponding to $H-3$ in the thione 16c. In this experiment, only one dithiin was observed probably due to fast isomerization of diradical 11(i) to carbene 11(iv) avoiding the cyclization of diradical 11(i) to the thiirene intermediate.

FVT of 4-phenyl-5-(heteroaryl)-1,2,3-thiadiazole (17). Thermolysis of 1-(4-phenyl-1,2,3thiadiazol-5-yl)-1H-1,2,3-benzotriazole (17) was carried out between $200-300{ }^{\circ} \mathrm{C}$ (Scheme 5). Nitrogen extrusion and subsequent rearrangements gave the zwitterion product $\mathbf{1 8}$ in good yield $(18-72 \%)$. It is proposed that this reaction proceeds through the mechanism shown in Scheme 5. Formation of the diradical 17(iii) is followed by opening of the benzotriazole ring, a transannular cyclization then gives the final product 18. This type of ring contraction has been observed in the thermolysis of eight and nine-membered rings. ${ }^{6,10}$ It should be noted that the zwitterion 18 was also obtained as the main product in the thermal reactions of thiadiazole 17 using DMF at 150$170^{\circ} \mathrm{C} .{ }^{9 \mathrm{~b}}$ In that case the structure of this compound was determined by single crystal X-ray analysis and the yield of those reactions were about $39 \%$.

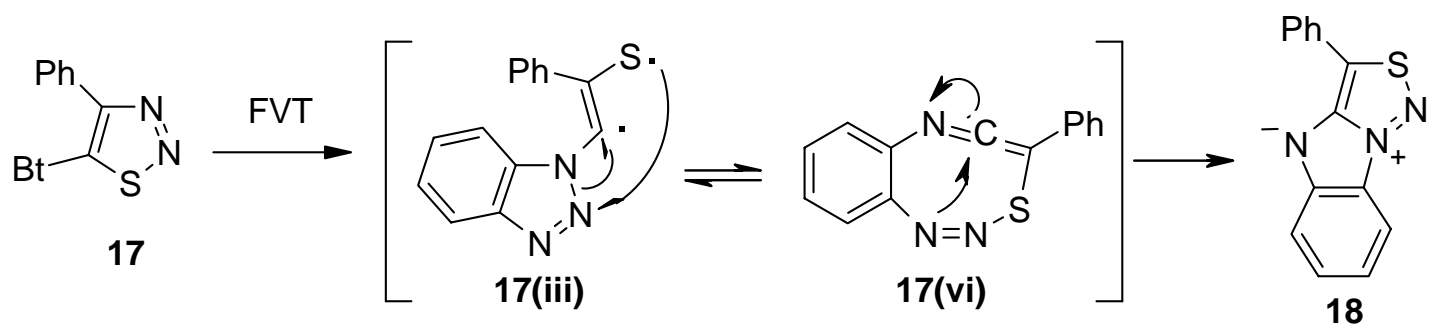

FVT of 17. Bt = 1-benzotriazolyl

\begin{tabular}{lccc}
\hline $\mathrm{T}\left({ }^{\circ} \mathrm{C}\right)$ & $\% \mathbf{1 7}$ & $\mathbf{1 8} \mathrm{Y}(\%)$ & others $\mathrm{Y}(\%)^{\mathbf{a}}$ \\
\hline 200 & 74 & 18 & 8 \\
250 & 22 & 58 & 20 \\
300 & 2 & 72 & 26 \\
& \\
& & \\
& &
\end{tabular}

\section{Scheme 5}




\section{Conclusions}

In summary, we have shown that substituted thiadiazoles undergo a nitrogen extrusion reaction at fairly low temperatures $\left(150{ }^{\circ} \mathrm{C}\right.$ for 5-aryloxythiadiazoles $)$ under FVT conditions to give diradical intermediates that can undergo different rearrangements depending upon the substituents present. Products formed are in accordance with the earlier observations such as involvement of thiirenes, and coupling of diradicals. Our observations further demonstrate the formation of products via insertion of sulfur-centered radicals or thioketenes into a $\mathrm{C}-\mathrm{H}$ bond of the aromatic ring and transannular cyclizations involving heteroaryl rings.

\section{Experimental Section}

General Procedures. Thermolysis was carried out in a glass FVT equipment using a GAYNOR PRDH temperature controller and a Thermolyne 21100 tube furnace, $30 \mathrm{~cm}$ (length) and $1 \mathrm{~cm}$ (diameter). Oxygen free dry nitrogen or a mixture of nitrogen/toluene was used as carrier gas. Flow rates of carrier gas were between $0.1-0.09 \mathrm{ml} \mathrm{sec}^{-1}$. Samples to be pyrolyzed were $20-80$ $\mathrm{mg}$. Contact times were around $10^{-2} \mathrm{sec}$ and the pressure from $0.02-0.01$ Torr (with and without carrier gas) was measured with a McLeod manometer. Products were trapped at liquid air temperature, extracted with solvent and submitted to different analyses or separation techniques. Quantification of products by ${ }^{1} \mathrm{H}-\mathrm{NMR}$ spectroscopy was made using exact amounts of 2,4dinitro-chlorobenzene as an internal standard. Gas chromatography/mass spectrometry (GC/MS) analyses were performed with a Perkin Elmer Q-Mass 910 spectrometer equipped with an SE-30 column, using helium as eluent at a flow rate of $1 \mathrm{ml} / \mathrm{min}$ and a heating rate of $40{ }^{\circ} \mathrm{C}$ for $5 \mathrm{~min}$ and $400{ }^{\circ} \mathrm{C}$ to $280{ }^{\circ} \mathrm{C}$ for $40 \mathrm{~min} .{ }^{1} \mathrm{H}-\mathrm{NMR}$ and ${ }^{13} \mathrm{C}$-NMR spectra were recorded in $\mathrm{CCl}_{4}$ solution with a capillary tube filled with acetone- $d_{6}$ inside the NMR tube or $\mathrm{CDCl}_{3}$ using a Bruker $200 \mathrm{FT}$ spectrometer (working at 200 and $75 \mathrm{MHz}$ respectively). Chemical shifts are reported in parts per million ( $\mathrm{ppm}$ ) downfield from TMS. Elemental analyses were performed on a Perkin-Elmer 240C microanalyser.

2,5-Diphenyl-3,6-dipropyl-1,4-dithiine (3) and 2,6-Diphenyl-3,5-dipropyl-1,4-dithiine (4). The product was a mixture of 3 and $4 .{ }^{1} \mathrm{H}-\mathrm{NMR} \delta 0.88(\mathrm{t}, J=7 \mathrm{~Hz}, 6 \mathrm{H}), 0.91(\mathrm{t}, J=7 \mathrm{~Hz}, 6 \mathrm{H})$, 1.40 (q, $J=7 \mathrm{~Hz}, 4 \mathrm{H}), 1.42$ (q, $J=7 \mathrm{~Hz}, 4 \mathrm{H}), 2.32$ (t, $J=7 \mathrm{~Hz}, 4 \mathrm{H}), 2.35$ (t, $J=7 \mathrm{~Hz}, 4 \mathrm{H}), 7.18$ $7.40(\mathrm{~m}, 20 \mathrm{H}) .{ }^{13} \mathrm{C}-\mathrm{NMR} \delta 13.6,13.9,21.1,21.2,34.0,34.2,112.4,113.4,126.7,126.8,126.9$, 127.3, 128.5, 128.5, 128.7, 128.8, 137.5, 141.4. MS (m/z) $352\left(17 \%, \mathrm{M}^{+}\right), 323$ (19), 176 (30), 147 (100), 103 (58). Anal. Calcd for $\mathrm{C}_{22} \mathrm{H}_{24} \mathrm{~S}_{2}: \mathrm{C}, 74.95 ; \mathrm{H}, 6.86 ; \mathrm{S}, 18.19$. Found: C, 74.87; H, $6.63 ; \mathrm{S}, 18.32$.

Trimer 5(a). White amorphous crystals; $\mathrm{mp} 130{ }^{\circ} \mathrm{C}(\mathrm{dec}$.$) . MS (m/z) 529\left(4 \%, \mathrm{M}^{+}\right), 497$ (7), 468 (7), 353 (23), 324 (16), 208 (5), 177 (37), 103 (86). IR $1080 \mathrm{~cm}^{-1}, v \mathrm{C}=\mathrm{S}$. 
2-Phenyl-4H-thiochromene (6) and 3-Phenyl-4H-thiochromene (7). The product was a mixture of 6 and 7. ${ }^{1} \mathrm{H}-\mathrm{NMR} \delta 3.75(\mathrm{~s}, 2 \mathrm{H}), 3.78(\mathrm{~d}, J=3 \mathrm{~Hz}, 2 \mathrm{H}), 6.95-7.32(\mathrm{~m}, 20 \mathrm{H}) .{ }^{13} \mathrm{C}-$ NMR $\delta 38.0,40.3,124.8,126.0,126.4,126.4,126.9,127.8,127.8,128.0,128.1,128.1,128.5$, $128.7,130.6,131.0,132.3,133.1,133.6,133.6,133.8,134.5,141.8,142.5 . \mathrm{MS}(\mathrm{m} / \mathrm{z}) 224(21 \%$, $\mathrm{M}^{+}$), 191 (62), 147 (9), 91 (100). Anal. Calcd for $\mathrm{C}_{15} \mathrm{H}_{12} \mathrm{~S}: \mathrm{C}, 80.31$; H, 5.39. Found: C, 80.11; H, 5.68 .

2-(4-Methylphenoxy)benzothiophene (9a). Yellow oil. ${ }^{1} \mathrm{H}-\mathrm{NMR} \delta 2.44$ (s, 3H), 6.93-7.32 (m, 9H). ${ }^{13} \mathrm{C}-\mathrm{NMR} \delta$ 21.4, 111.2, 120.5, 127.6, 127.9, 128.1, 129.1, 130.8, 132.8, 141.6, 145.9, 154.6, 158.4. MS (m/z) $240\left(26 \%, \mathrm{M}^{+}\right), 133$ (7), 107 (35), 91 (100). Anal. Calcd for $\mathrm{C}_{15} \mathrm{H}_{12} \mathrm{OS}$ : C, 74.97; H, 5.03; S, 13.34. Found: C, 75.01; H, 4.80; S, 13.92.

2-(4-Methoxyphenoxy)benzothiophene (9b). Yellow oil. ${ }^{1} \mathrm{H}-\mathrm{NMR} \delta 3.82(\mathrm{~s}, 3 \mathrm{H}), 6.86$ (d, $J=$ $9 \mathrm{~Hz}, 2 \mathrm{H}), 6.95-7.49(\mathrm{~m}, 7 \mathrm{H}) .{ }^{13} \mathrm{C}-\mathrm{NMR} \delta 52.3,94.9,114.3,120.1,122.2,122.5,124.6,125.1$, 137.7, 139.5, 152.5, 156.0, 160.2. MS (m/z): $256\left(5 \%, \mathrm{M}^{+}\right), 149$ (100), 121 (5), 104 (21). Anal. Calcd for $\mathrm{C}_{15} \mathrm{H}_{12} \mathrm{O}_{2} \mathrm{~S}$ : C, 70.29; H, 4.72; S, 12.51. Found: C, 70.15; H, 4.18; S, 12.61 .

2,5-Bis-[(4-methylphenyl)thio]-3,6-diphenyl-1,4-dithiin (12a). Yellow micro crystals; mp 147-

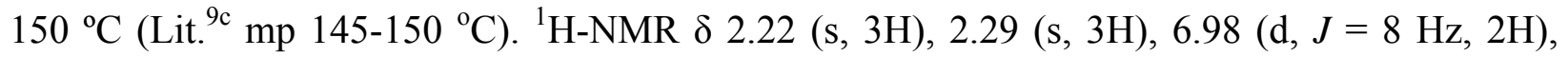
7.00-7.30 (m, 12H), 7.46-7.53 (m, 4H). ${ }^{13} \mathrm{C}-\mathrm{NMR} \delta 20.5,21.3,111.0,117.3,127.1,127.5,127.5$, $128.5,128.9,129.1,129.3,130.0,130.2,131.1,131.5,131.5,135.3,137.7,139.3,140.9$.

2,5-Bis[(4-chlorophenyl)thio]-3,6-diphenyl-1,4-dithiin (12b). Yellow micro crystals; mp 157$159{ }^{\circ} \mathrm{C} .{ }^{1} \mathrm{H}-\mathrm{NMR} \delta 7.30-7.91(\mathrm{~m}, 18 \mathrm{H}) .{ }^{13} \mathrm{C}-\mathrm{NMR} \delta 120.5,128.0,129.1,129.5,130.2,133.1$, 135.0, 135.3, 142.7, 150.1. Anal. Calcd for $\mathrm{C}_{28} \mathrm{H}_{18} \mathrm{Cl}_{2} \mathrm{~S}_{4}$ : C, 60.75; H, 3.28. Found: C, 60.42; H, 3.59 .

2,5-Bis-[3,6-dimethylphenyl)thio]-3,6-diphenyl-1,4-dithiin (12c). White micro crystals; mp: 148-151 ${ }^{\circ} \mathrm{C}$ (Lit. ${ }^{9 \mathrm{c}} \mathrm{mp} 150-152{ }^{\circ} \mathrm{C}$ ). ${ }^{1} \mathrm{H}-\mathrm{NMR} \delta 2.23$ (s, 6H), 2.35 (s, 6H), 6.85-7.55 (m, 16H). ${ }^{13} \mathrm{C}-\mathrm{NMR} \delta 19.5,21.0,126.3,127.1,127.5,127.5,127.7,128.5,128.9,128.9,129.1,129.3$, $130.0,130.5,130.5,131.1,133.7,134.0,135.5,137.1,139.3$.

5-Chloro-3-phenyl-1-benzothiophene-2-thiol (13b). Yellow oil. ${ }^{1} \mathrm{H}-\mathrm{NMR} \delta 3.55$ (s, 1H). 7.01$7.70(\mathrm{~m}, 8 \mathrm{H}) .{ }^{13} \mathrm{C}-\mathrm{NMR} \delta 119.9,123.3,127.0,127.9,128.4,128.5,129.0,132.7,134.0,136.2$, 137.5, 142.4. MS (m/z) $278 / 276\left(\mathrm{M}^{+}, 34 / 100 \%\right), 240$ (65), 208 (16), 195 (21), 120 (40). Anal. Calcd for $\mathrm{C}_{14} \mathrm{H}_{9} \mathrm{ClS}_{2}$ : C, 60.75; H, 3.28. Found: C, 60.38; H, 3.01.

4,7-Dimethyl-3-phenyl-1-benzothiophene-2-thiol (13c). Yellow oil. ${ }^{1} \mathrm{H}-\mathrm{NMR} \delta 2.48(\mathrm{~s}, 3 \mathrm{H})$, $2.92(\mathrm{~s}, 3 \mathrm{H}), 3.78(\mathrm{~s}, 1 \mathrm{H}), 7.04-7.62(\mathrm{~m}, 7 \mathrm{H}) .{ }^{13} \mathrm{C}-\mathrm{NMR} \delta 19.7,21.4,118.8,124.9,127.8,128.5$, 128.9, 129.4, 130.3, 131.7, 134.4, 135.1, 137.3, 139.3. MS (m/z) $270\left(100 \%, \mathrm{M}^{+}\right), 254$ (32), 237 (57), 221 (22). Anal. Calcd for $\mathrm{C}_{16} \mathrm{H}_{14} \mathrm{~S}_{2}$ : C, 71.07; H, 5.22. Found: C, 70.71; H, 5.59.

5-Methyl-2-[(5-methyl-3-phenylbenzothien-2-yl)dithio]-3-phenylbenzothiophene

Yellow micro crystals; mp $188-191{ }^{\circ} \mathrm{C} .{ }^{1} \mathrm{H}-\mathrm{NMR} \delta 2.37,(\mathrm{~s}, 6 \mathrm{H}), 6.92-7.67(\mathrm{~m}, 16 \mathrm{H}) .{ }^{13} \mathrm{C}-\mathrm{NMR}$ $\delta 21.3,119.3,125.2,127.5,127.9,128.1,133.4,135.8,137.6,141.6,1482,154.4 . \mathrm{MS}(\mathrm{m} / \mathrm{z}) 510$ $\left(5 \%, \mathrm{M}^{+}\right), 256$ (100), 240 (50), 224 (35). Anal. Calcd for $\mathrm{C}_{16} \mathrm{H}_{14} \mathrm{~S}_{2}$ : C, 71.07; H, 5.22. Found: C, $70.71 ; \mathrm{H}, 5.59$. 
5-Chloro-2-[(5-chloro-3-phenylbenzothien-2-yl)dithio]-3-phenylbenzothiophene

(14b). Yellow micro crystals; mp $198-200{ }^{\circ} \mathrm{C} .{ }^{1} \mathrm{H}-\mathrm{NMR} \delta$ 7.10-7.63 (m, 16H). ${ }^{13} \mathrm{C}-\mathrm{NMR} \delta 119.5,123.5$, 127.2, 127.8, 127.9, 129.5, 133.6, 134.8, 141.6, 142.1, 149.2, 152.4. MS (m/z) 554/552/550 $\left(2 / 9 / 12 \%, \mathrm{M}^{+}\right), 274 / 276(100 / 35 \%), 275 / 277(13 / 7), 240(60), 208$ (10), 195 (15), 120 (29). Anal. Calcd for $\mathrm{C}_{28} \mathrm{H}_{16} \mathrm{Cl}_{2} \mathrm{~S}_{4}: \mathrm{C}, 60.97 ; \mathrm{H}, 2.92$. Found: $\mathrm{C}, 61.25 ; \mathrm{H}, 2.89$.

2-[(4,7-Dimethyl-1-benzothien-2-yl)dithio]-4,7-dimethyl-1-benzothiophene (14c). Yellow micro crystals; mp 190-192 ${ }^{\circ} \mathrm{C} .{ }^{1} \mathrm{H}-\mathrm{NMR} \delta 2.49$ (s, 6H), 2.75 (s, 6H), 6.85-7.00 (m, 14H). ${ }^{13} \mathrm{C}-$ NMR $\delta 20.3,21.4,125.3,127.5,127.6,128.3,129.0,129.3,130.5,133.3,133.8,139.6,149.7$, 151.8. MS (m/z) $538\left(21 \%, \mathrm{M}^{+}\right), 270(92), 255$ (100), 222 (12), 121 (7). Anal. Calcd for $\mathrm{C}_{32} \mathrm{H}_{26} \mathrm{~S}_{4}: \mathrm{C}, 71.33 ; \mathrm{H}, 4.86$. Found: C, 71.39; H, 4.52.

p-Tolyl disulfide (15a) (CAS 103-19-5). This compound was identified by ${ }^{1} \mathrm{H}-\mathrm{NMR}$ and GC/MS using authentic samples for testing. ${ }^{1} \mathrm{H}-\mathrm{NMR} \delta 2.31$ (s, 6H), $7.09(\mathrm{~d}, J=8 \mathrm{~Hz}, 4 \mathrm{H}), 7.35$ (d, $J=8 \mathrm{~Hz}, 4 \mathrm{H}) . \mathrm{GC} / \mathrm{MS} \mathrm{t}_{\mathrm{r}}=21.75 \mathrm{~min}$. MS (m/z) $246\left(29 \%, \mathrm{M}^{+}\right), 123(100), 108$ (70).

p-Chlorophenyl disulfide (15b) (CAS 1142-19-4). This compound was identified by ${ }^{1} \mathrm{H}-\mathrm{NMR}$ and GC/MS using authentic samples for testing. ${ }^{1} \mathrm{H}-\mathrm{NMR} \delta 7.38,7.30(\mathrm{AB}, J=8 \mathrm{~Hz}, 4 \mathrm{H})$.

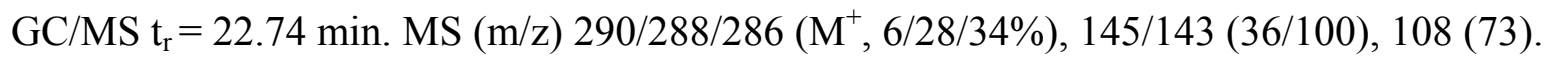

2,5-Dimethylphenyl disulfide (15c). This compound was identified from the reaction mixture by ${ }^{1} \mathrm{H}-\mathrm{NMR}$ and GC/MS. ${ }^{1} \mathrm{H}-\mathrm{NMR} \delta 2.28(\mathrm{~s}, 6 \mathrm{H}), 2.38(\mathrm{~s}, 6 \mathrm{H}), 6.80-7.10(\mathrm{~m}, 6 \mathrm{H}) . \mathrm{CG} / \mathrm{MS} \mathrm{t}_{\mathrm{r}}=$ $23.27 \mathrm{~min}$. MS (m/z) $288\left(39 \%, \mathrm{M}^{+}\right), 137$ (100), 105 (16), 91 (47), 77 (34).

4-Phenyl-6H-[1,2,3]thiadiazolo[3,4-a]benzimidazol-1-ium-6-ide (18). Red-orange micro crystals; mp 132-133 ${ }^{\circ} \mathrm{C}$ (Lit. ${ }^{9 b} \mathrm{mp} 137-138{ }^{\circ} \mathrm{C}$ ). ${ }^{1} \mathrm{H}-\mathrm{NMR} \delta$ 7.26-7.36 (m, 1H), 7.41-7.61 (m, 4H), $7.98(\mathrm{~d}, J=8 \mathrm{~Hz}, 1 \mathrm{H}), 8.15(\mathrm{~d}, J=8 \mathrm{~Hz}, 1 \mathrm{H}), 8.27$ (d, $J=8 \mathrm{~Hz}, 2 \mathrm{H}) .{ }^{13} \mathrm{C}-\mathrm{NMR} \delta 112.9$, 120.5, 121.0, 127.0, 128.0, 128.6, 129.6, 130.3, 131.1, 132.5, 151.6, 154.1.

\section{References}

1. (a) Thomas, E. W. In Comprehensive Heterocyclic Chemistry II; Katritzky, A. R.; Rees, C. W.; Scriven, E. F. V., Eds.; Pergamon Press: Oxford, 1996; Vol. 4, p 289. (b) Thomas, E. W. In Comprehensive Heterocyclic Chemistry II; Katritzky, A. R.; Rees, C. W.; Eds.; Pergamon Press: Oxford, 1984; Vol. 6, p 447.

2. Murai, H.; Torres, M.; Strausz, O. P. J. Am. Chem. Soc. 1976, 101, 3976.

3. (a) Krantz, A.; Laureni, J. J. Am. Chem Soc. 1981, 103, 486. (b) Wentrup, C. Adv. Heterocycl. Chem. 1981, 28, 231. (c) Torres, M.; Clement, A.; J. E.; Gunning, H. E.; Strausz, O. P. J. Org. Chem. 1978, 43, 2490. (d) Krantz, A.; Laureni, J. J. Am.Chem. Soc. 1977, 99, 4842.

4. (a) Jorgensen, T.; Pederson, C. T.; Flammang, R.; Wentrup, C. J. Chem. Soc., Perkin Trans 2 1997, 173. (b) Schaumann, E.; Ehlers, J.; Mrotzek, H. Liebigs Ann. Chem. 1979, 1734. (c) Seybold, G.; Heibl, C. Chem. Ber. 1977, 110, 1225. (d) Seybold, G.; Heibl, C. Angew. Chem. Int. Ed. 1975, 14, 248. 
5. Schaumann, E. Tetrahedron 1988, 44, 1827.

6. Yranzo, G. I.; Elguero, J.; Flammang, R.; Wentrup, C. Eur. J. Org. Chem. 2001, 2209.

7. (a) Buhl, H.; Timm, U.; Meier, H. Chem. Ber. 1979, 112, 3728. (b) Buhl, H.; Seitz, B.; Meier, H. Tetrhedron 1977, 33, 449. (c) Zeller, K.-P.; Meier, H.; Muller, E. Tetrahedron Lett. 1971, 42, 537.

8. (a) Voets, M.; Smet, M.; Dehaen, W. J. Chem. Soc., Perkin Trans. 1 1999, 1473. (b) Ganjian, I. J. Heterocycl. Chem. 1990, 2037. (c) Raap, R.; Micetich, R. G. Can. J. Chem. 1968, 1057.

9. (a) Katritzky, A. R.; Nikonov, G. N.; Moyano, E. L.; Akhmedov, N. G.; Steel, P. J. ARKIVOC 2003, (vii), 121. (b) Katritzky, A. R.; Nikonov, G. N.; Tymoshenko, D. O.; Steel, P. J. Heterocycles 2002, 58, 311. (c) Katritzky, A. R.; Nikonov, G. N.; Tymoshenko, D. O.; Moyano, E. L.; Steel, P. J. Heterocycles 2002, 57, 483. (d) Katritzky, A. R.; Tymoshenko, D. O.; Nikonov, G. N. J. Org. Chem. 2001, 66, 4045.

10. Banciu, M. D.; Popescu, A.; Simion, A.; Draghici, C.; Mangra, C.; Mihaiescu, D.; Pocol, M. J. Anal. Appl. Pyrolysis 1999, 48, 129. 